\title{
Towards the complete picture: computational strategies to identify MS-Imaging derived molecular fingerprints associated with inflammatory cell components
}

Chang Lu ${ }^{1}$, Pieter Goossens ${ }^{1}$, Jianhua Cao $^{3}$, Joel Karel ${ }^{2}$, Evgeni Smirnov², Marion Gijbels ${ }^{1}$, Benjamin Balluff ${ }^{3}$, Ron Heeren ${ }^{3}$, Erik A.L. Biessen ${ }^{1}$

${ }^{1}$ Cardiovascular Research Institute Maastricht (CARIM), Maastricht University, Maastricht, Netherlands; ${ }^{2}$ Department of Data Sciences and Knowledge Engineering (DKE), Maastricht University, Maastricht, Netherlands; ${ }^{3}$ Maastricht Multimodal Molecular Imaging Institute (M4I), Maastricht University, Maastricht, Netherlands;

\section{Background and Objective}

Macrophages can adapt to stimuli in their direct environment, adopting a functional phenotypic spectrum throughout the disease course of atherosclerosis. While this suggests significant plaque macrophage heterogeneity, this has thus far only been poorly described, largely due to technical constraints. Here we present a new strategy to dissect macrophage heterogeneity in its molecular context at high spatial resolution.

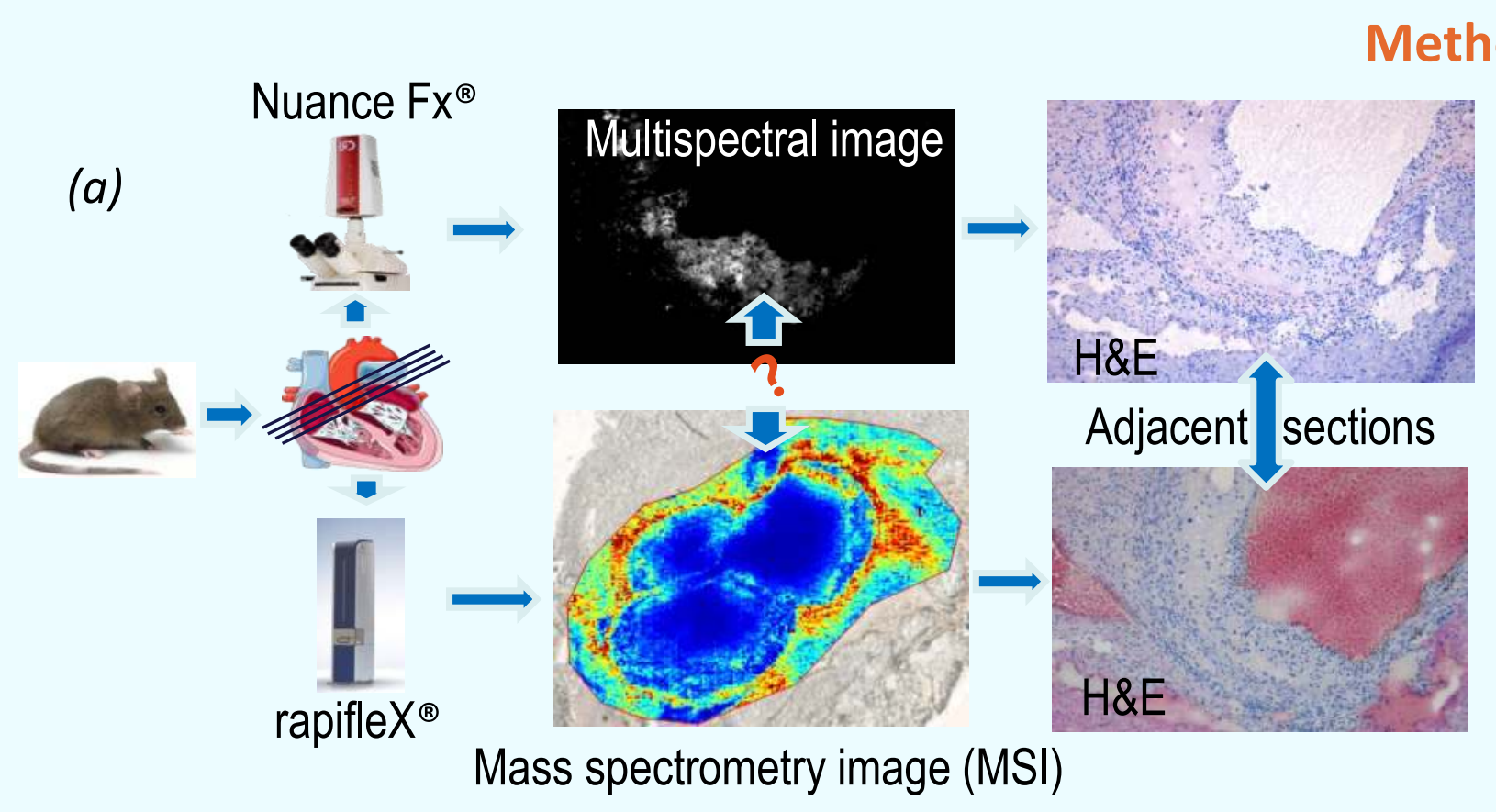

Fig. 1: (a) Data collection. (b) Pipeline.

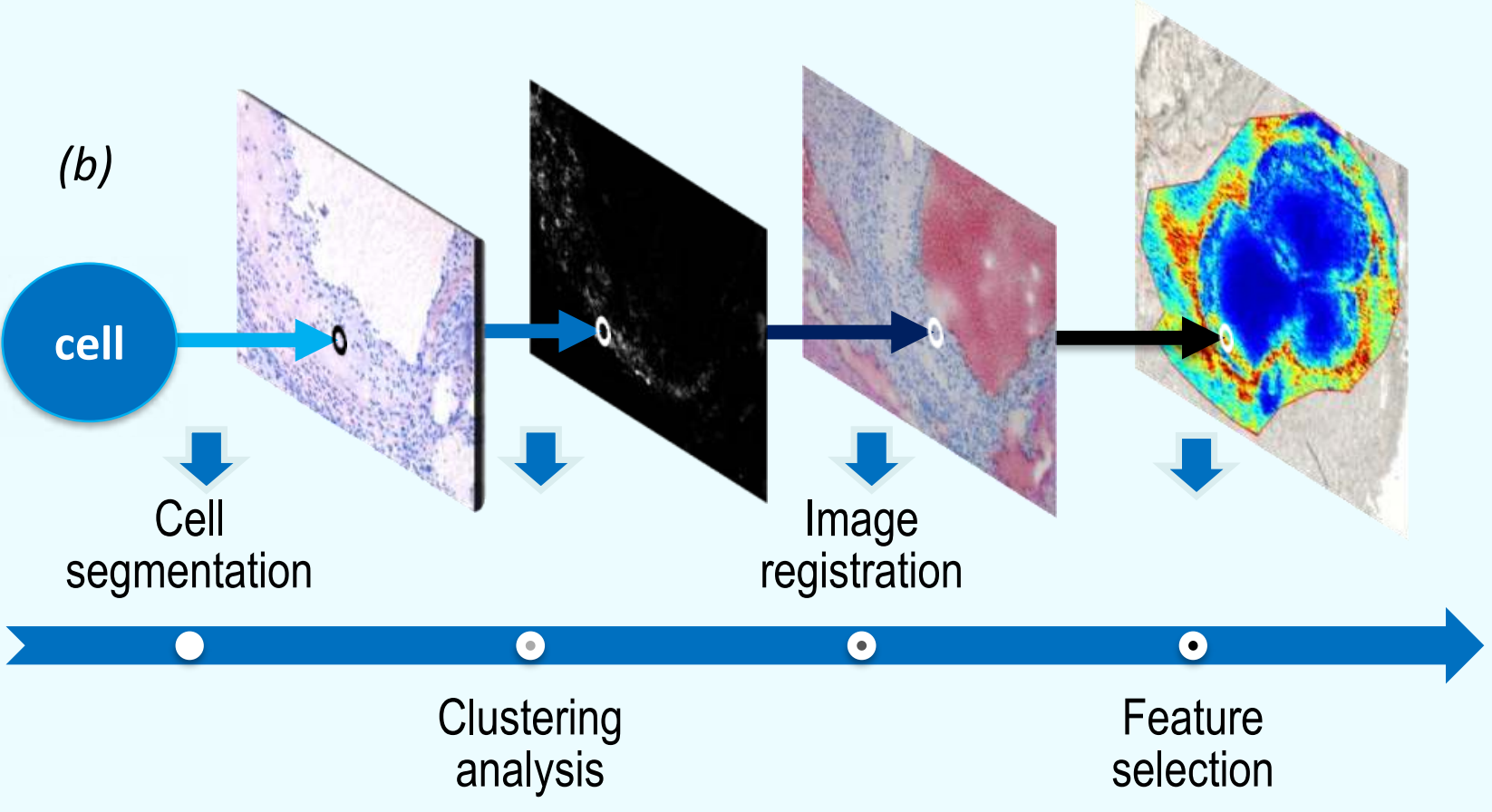

Results

\section{Clustering analysis}

Overlaying a cell segmentation mask on 12 phenotype-defining fluorescent channels resulted in a matrix of mean fluorescent intensities of these markers for each individual cell. Cell segments were grouped into 30 clusters, using a cosine similarity-based Kmedoids approach.

(a)

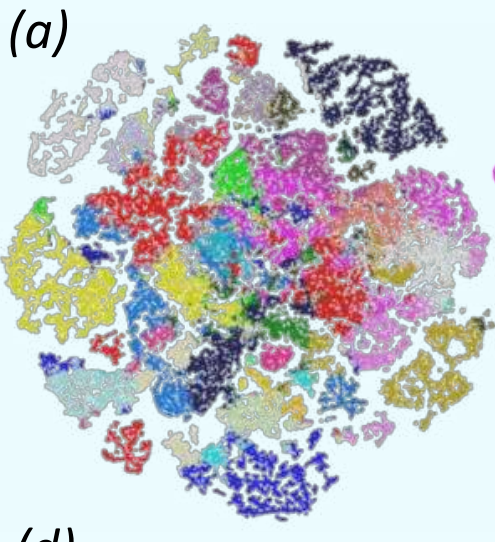

(d)

(d)

$1 \%$

\&

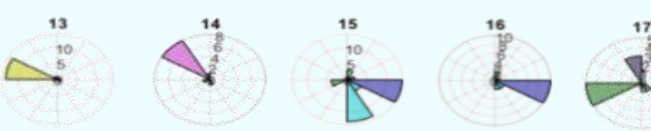

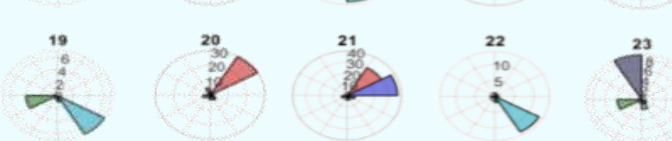

$i^{25} \overbrace{}^{20} \overbrace{}^{27} \overbrace{}^{20}$

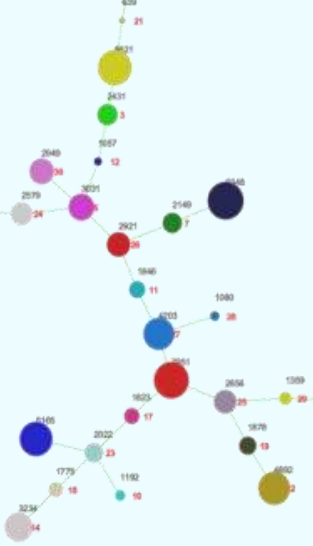

Fig. 2: Visualization of clustering results shown on $\operatorname{tSNE}(a)$, minimal spanning tree (b), k-nearest neighbor network ((c), $k=3)$, histogram on each channel (d), violin plot (e). Each cluster can also be overlaid on the H\&E image for pathological analysis (f).

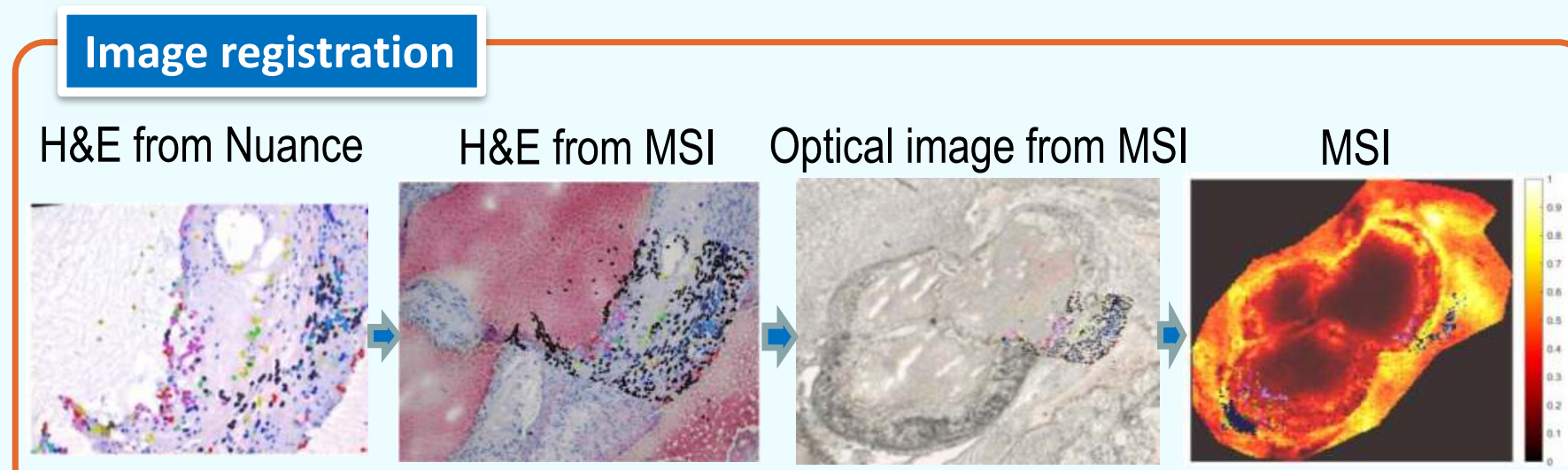

Fig. 3: Two sections were aligned by a series of (rigid and elastic) image registration algorithms, mapping the positions of the identified macrophage clusters on the MSI dataset to label each MS pixel a certain population.

Feature selection

Fig. 4: (a) Several of the 30 cell clusters were combined based on domain knowledge and hierarchical clustering

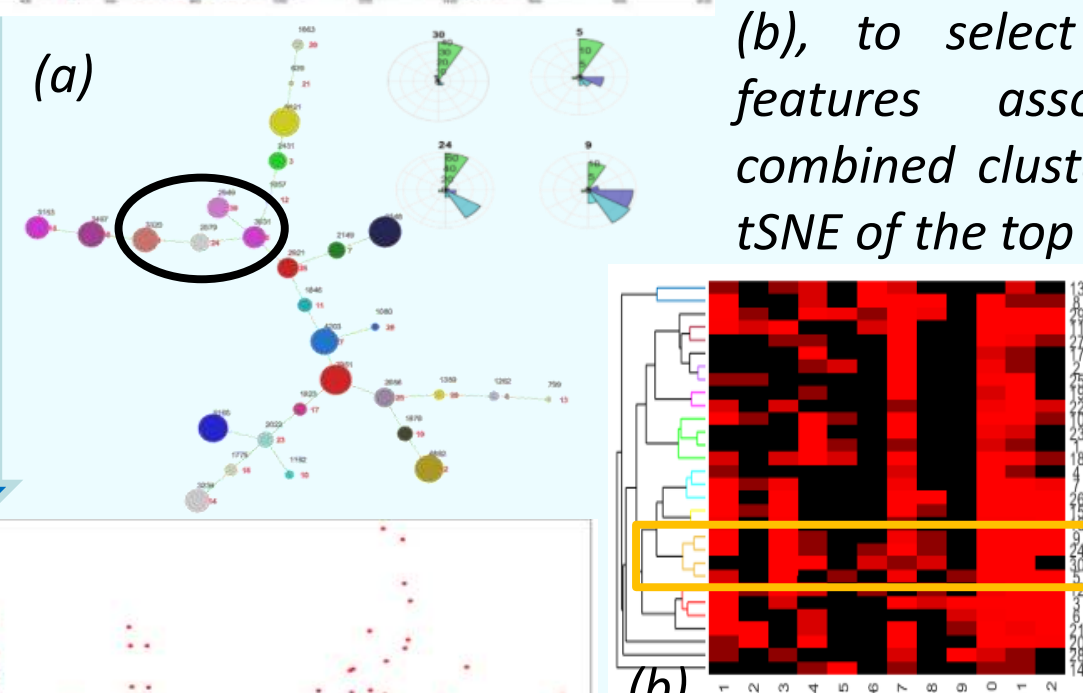

(b)

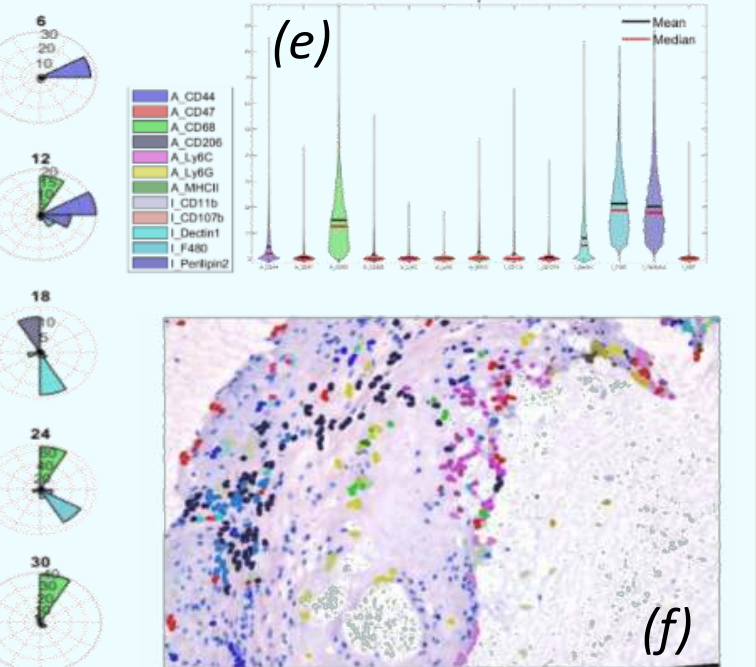

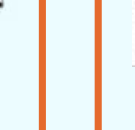

\begin{tabular}{|c|c|c|c|c|c|c|}
\hline$M / Z$ & 788.6497 & 794.6498 & 792.6498 & 574.5243 & 762.6090 & 789.6497 \\
\hline Lipids & $\begin{array}{l}\text { C45H91N } \\
\text { O7P }\end{array}$ & $\begin{array}{l}\text { C47H88N } \\
08\end{array}$ & $\begin{array}{l}\text { C51H86N } \\
\text { O5 }\end{array}$ & $\begin{array}{l}\mathrm{C} 35 \mathrm{H} 71 \mathrm{~N} \\
\mathrm{O} 2[37] \mathrm{Cl}\end{array}$ & $\begin{array}{l}\mathrm{C} 42 \mathrm{H} 85 \mathrm{~N} \\
\text { O8P }\end{array}$ & $\begin{array}{l}\text { C44H90N } \\
207 P\end{array}$ \\
\hline
\end{tabular}

\section{Conclusion}

We have developed an innovative new approach to define not only cell heterogeneity, but also to link this to the microenvironment. The methodology is also applicable to other cell types and tissue and may lead to a breakthrough in linking molecular context to cellular phenotype and function, in healthy and diseased tissues.

$\begin{array}{ccc}\text { Correspondence to: } & \text { Department of Pathology } & \text { Maastricht University } \\ \text { Erik A.L. Biessen } & \mathrm{T}+31433877675 & \text { P.O. Box 616 } \\ \text { erik.biessen@mumc.nl } & \mathrm{F}+31433876613 & 6200 \mathrm{MD} \text { Maastricht, The } \\ \text { www.carimmaastricht.nl } & \text { Netherlands }\end{array}$

